\title{
On Spectral Homotopy Analysis Method for Solving Linear Volterra and Fredholm Integrodifferential Equations
}

\author{
Z. Pashazadeh Atabakan, A. Kılıçman, and A. Kazemi Nasab \\ Department of Mathematics, Universiti Putra Malaysia (UPM), Selangor, 43400 Serdang, Malaysia \\ Correspondence should be addressed to A. Kılıçman, akilicman@putra.upm.edu.my
}

Received 12 June 2012; Revised 19 September 2012; Accepted 4 October 2012

Academic Editor: Lishan Liu

Copyright (C) 2012 Z. Pashazadeh Atabakan et al. This is an open access article distributed under the Creative Commons Attribution License, which permits unrestricted use, distribution, and reproduction in any medium, provided the original work is properly cited.

\begin{abstract}
A modification of homotopy analysis method (HAM) known as spectral homotopy analysis method (SHAM) is proposed to solve linear Volterra integrodifferential equations. Some examples are given in order to test the efficiency and the accuracy of the proposed method. The SHAM results show that the proposed approach is quite reasonable when compared to SHAM results and exact solutions.
\end{abstract}

\section{Introduction}

Functional equations such as partial differential equations, integral and integrodifferential equations and others are widely employed to model complex real-life problems. Many physical events in different fields of sciences and engineering have been formulated using integrodifferential equations $[1,2]$. Integrodifferential equations are usually difficult to solve analytically so there is a need to obtain an efficient approximate solution [3, 4]. Homotopy analysis method (HAM) was first proposed by Liao in [5]. it is based on the homotopy concept in topology for solving nonlinear differential equations. Unlike the traditional perturbation methods like Lyapunov's artificial small parameter method [6], Adomian decomposition method [7-10] and the $\delta$-expansion method [11], which are the special cases of HAM, this approach does not need a small perturbation parameter. In the HAM, original nonlinear problem is converted to infinite number of linear problems without using the perturbation techniques [12]. Homotopy analysis method is more powerful than traditional perturbation methods since it is applicable for solving problems with strong nonlinearity [13-15], even if they do not have any small or large parameters. 
We can adjust the convergence region and the rate of approximation series to give us freedom to use different base function to approximate a nonlinear problem. He in [16] proposed the so called Homotopy perturbation method (HPM). Later it was pointed by Hayat et al. [17] that HPM is only a special case of the HAM $(h=-1)$. Many nonlinear problems were solved by Homotopy perturbation method [18, 19]. The HPM does not provide us with convergent series solution for strongly nonlinear problems as was indicated by Abbasbandy in [20] using a simple problem in which the physical parameters were large. In 2010, Motsa et al. [21] employed Chebyshev polynomials to solve higher-order deformation equation and called his approach spectral homotopy analysis method (SHAM). The spectral homotopy analysis method has been used only for solving partial and ordinary differential equations [22, 23]. Motsa et al. [21-23] found that the Spectral homotopy analysis method is more efficient than the homotopy analysis method as it does not depend on the rule of solution expression and the rule of ergodicity. This method is more flexible than homotopy analysis method since it allows for a wider range of linear and nonlinear operators and one is not restricted to use the method of higher order differential mapping for solving boundary value problems in bounded domains, unlike the homotopy analysis method. The range of admissible $h$ values is much wider in spectral homotopy analysis method than in homotopy analysis method. The main restriction of HAM in solving integral equations is to choose the best initial guess as the series solution be convergent. In SHAM the initial approximation is taken to be the solution of the nonhomogeneous linear part of the given equation.

In this paper, we propose spectral homotopy analysis method (SHAM) to solve linear Volterra and Fredholm type of integrodifferential equations. Volterra integrodifferential equation is given by

$$
\begin{gathered}
a_{1}(x) u^{\prime \prime}(x)+a_{2}(x) u^{\prime}(x)+a_{3}(x) u(x)=g(x)+\int_{-1}^{x} k(x, t) u(t) d t \\
u(-1)=0, \quad u(1)=0 .
\end{gathered}
$$

The paper is organized in the following way. Section 2 included a brief introduction in homotopy analysis method. Spectral homotopy analysis method for solving integral equations is presented in Section 3. We then propose the way to calculate $\mathbf{S}_{m-1}$ which is needed to obtain $\mathbf{Y}_{m}$ in Section 4. Numerical examples are presented in Section 5. In Section 6, concluding remarks are given.

\section{Homotopy Analysis Solution}

In this section, we give a brief introduction to HAM. We consider the following differential equation in a general form:

$$
N[u(\tau)]=0,
$$

where $N$ is nonlinear operator, $\tau$ denotes independent variables and $u(\tau)$ is an unknown function, respectively. For simplicity we disregard all initial and all boundary conditions 
which can be dealt in similar way. The so-called zero-order deformation equation was constructed by Liao as follows:

$$
(1-p) L\left[\phi(\tau ; p)-u_{0}(\tau)\right]=p h H(\tau)(N[\phi(\tau ; p)])
$$

where $p \in[0,1]$ is the embedding parameter and, $h$ is a nonzero convergence-parameter, $H(\tau)$ is an auxiliary function and $u_{0}(\tau)$ is called an initial guess of $u(\tau)$ and $\phi(\tau ; p)$ is an unknown function. In addition, $L$ is an auxiliary linear operator and $N$ is nonlinear operator as follows:

$$
L(\phi(x ; p))=a_{1}(x) \frac{\partial^{2} \phi(x ; p)}{\partial x^{2}}
$$

with the property $L\left(c_{1} t+c_{2}\right)=0$ where $c_{1}$ and $c_{2}$ are constants and

$$
N[\phi(x ; p)]=a_{1}(x) \frac{\partial^{2} \phi(x ; p)}{\partial x^{2}}+a_{2}(x) \frac{\partial \phi(x ; p)}{\partial x}+a_{3}(x) \phi(x ; p)-g(x)-\int_{-1}^{x} k(x, t) \phi(t) d t
$$

is a nonlinear operator. Obviously, when $p=0$ and $p=1$, it holds $\phi(\tau ; 0)=u_{0}(\tau)$ and $\phi(\tau ; 1)=$ $u(\tau)$. In this way, as $p$ increase from 0 to $1, \phi(\tau ; p)$ alter from initial guess $u_{0}(\tau)$ to the solution $u(\tau)$ and $\phi(\tau ; p)$ is expanded in Taylor series with respect to $p$ :

$$
\phi(\tau ; p)=u_{0}(\tau)+\sum_{m=1}^{+\infty} u_{m}(\tau) p^{m}
$$

where

$$
\begin{aligned}
& u_{m}(\tau)=D_{m}[\phi(\tau ; p)] \\
& D_{m} \phi=\left.\frac{1}{m !} \frac{\partial^{m}(\phi)}{\partial p^{m}}\right|_{p=0}
\end{aligned}
$$

The series (2.5) converges at $p=1$ if the auxiliary linear operator, the initial guess, the convergence parameter, and the auxiliary function are properly selected:

$$
\phi(\tau)=u_{0}(\tau)+\sum_{m=1}^{+\infty} u_{m}(\tau)
$$

The admissible and valid values of the convergence-parameter $h$ are found from the horizontal portion of the $h$-curves. Liao proved that $u(\tau)$ is one of the solutions of original nonlinear equation. As $H(\tau)=1$ so $(2.2)$ becomes

$$
(1-p) L\left[\phi(\tau ; p)-u_{0}(\tau)\right]=p h(N[\phi(\tau ; p)])
$$


Define the vector $u_{m}=\left\{u_{0}(\tau), u_{1}(\tau), \ldots, u_{m}(\tau)\right\}$. Operating on both sides with $D_{m}$, we have the so called $m$ th-order deformation equation

$$
L\left[u_{m}(\tau)-\chi_{m} u_{m-1}(\tau)\right]=h H(\tau) R_{m}\left(u_{m-1}(\tau)\right)
$$

where

$$
\begin{gathered}
R_{m}\left(u_{m-1}\right)=\left.\frac{1}{(m-1) !} \frac{\partial^{m-1} N[\phi(\tau ; p)]}{\partial p^{m-1}}\right|_{p=0}, \\
X_{m}= \begin{cases}0, & m \leq 1 \\
1, & \text { otherwise. }\end{cases}
\end{gathered}
$$

$u_{m}(\tau)$ for $m \geq 0$ is governed by the linear equation (2.10) and can be solved by symbolic computation software such as Maple, Matlab, and so on.

\section{Spectral Homotopy Analysis Solution}

Consider the second-order Volterra integrodifferential equation

$$
a_{1}(x) u^{\prime \prime}(x)+a_{2}(x) u^{\prime}(x)+a_{3}(x) u(x)=g(x)+\int_{-1}^{x} k(x, t) u(t) d t, \quad u(-1)=0, u(1)=0 .
$$

To obtain initial approximation we solve the following two-point boundary value problem:

$$
a_{1}(x) u_{0}^{\prime \prime}(x)+a_{2}(x) u_{0}^{\prime}(x)+a_{3}(x) u_{0}(x)=g(x)
$$

subject to boundary conditions

$$
u_{0}(-1)=0, \quad u_{0}(1)=0
$$

We use the Chebyshev pseudospectral method to solve (3.2). At first we approximate $u_{0}(\tau)$ by a truncated series of Chebyshev polynomial of the form

$$
u_{0}(\tau) \approx u_{0}^{N}\left(\tau_{j}\right)=\sum_{k=0}^{N} \widehat{u}_{k} T_{k}\left(\tau_{j}\right), \quad j=0, \ldots, N
$$

where $T_{k}$ is the $k$ th Chebyshev polynomials, $\widehat{u}_{k}$, are coefficients and $\tau_{0}, \tau_{1}, \ldots, \tau_{N}$ are GaussLobatto points which are the extrema of the $N$ th-order Chebyshev polynomial, defined by

$$
\tau_{j}=\cos \left(\frac{\pi j}{N}\right)
$$


Derivatives of the functions $y_{0}(\tau)$ at the collocation points are represented as

$$
\frac{d^{s} u_{0}\left(\tau_{k}\right)}{d \tau^{s}}=\sum_{j=0}^{N} D_{k j}^{s} u_{0}\left(\tau_{j}\right), \quad k=0, \ldots, N,
$$

where $s$ is the order of differentiation and $D$ is the Chebyshev spectral differentiation matrix. By following [24] we express the entries of the differentiation matrix $D$ as

$$
\begin{gathered}
D_{k j}=\left(\frac{-1}{2}\right) \frac{c_{k}}{c_{j}} \frac{(-1)^{k+j}}{\sin (\pi(j+k) / 2 N) \sin (\pi(j-k) / 2 N)}, \quad j \neq k, \\
D_{k j}=\left(\frac{-1}{2}\right) \frac{x_{k}}{\sin ^{2}(\pi k / N)}, \quad k \neq 0, N, k=j, \\
D_{00}=-D_{N N}=\frac{2 N^{2}+1}{6} .
\end{gathered}
$$

Substituting (3.4)-(3.6) into (3.2) results in

$$
\mathrm{AU}_{0}=\mathrm{G}
$$

subject to the boundary conditions

$$
u_{0}\left(\tau_{0}\right)=u_{0}\left(\tau_{N}\right)=0
$$

where

$$
\begin{aligned}
& \mathbf{A}=\mathbf{a}_{1} D^{2}+\mathbf{a}_{2} D+\mathbf{a}_{3}, \\
& \mathbf{U}_{0}=\left[u_{0}\left(\tau_{0}\right), u_{0}\left(\tau_{1}\right), \ldots, u_{0}\left(\tau_{N}\right)\right]^{T}, \\
& \mathbf{G}=\left[g\left(\tau_{0}\right), g\left(\tau_{1}\right), \ldots, g\left(\tau_{N}\right)\right]^{T}, \\
& \mathbf{a}_{r}=\operatorname{diag}\left(a_{r}\left(\tau_{0}\right), a_{r}\left(\tau_{1}\right), \ldots a_{r}\left(\tau_{N}\right)\right), \quad r=1,2,3 .
\end{aligned}
$$

To satisfy the boundary conditions we delete the first and the last rows and columns of A and the first and the last rows of $\mathbf{Y}_{0}$ and $\mathrm{G}$. The values of $u_{0}\left(\tau_{i}\right), i=0, \ldots, N$ are determined from the equation

$$
\mathbf{U}_{0}=\mathbf{A}^{-1} \mathbf{G}
$$

which is the initial approximation for the SHAM solution of the governing equation (3.1). The zeroth-order deformation equation is given by

$$
(1-p) L\left[\phi(\tau ; p) u_{0}(\tau)\right]=p h(N[\phi(\tau ; p)]-g(\tau))
$$


where

$$
L[\phi(\tau ; p)]=a_{1} \frac{\partial^{2} \phi}{\partial \tau^{2}}+a_{2} \frac{\partial \phi}{\partial \tau}+a_{3} \phi
$$

$h$ is the nonzero convergence controlling auxiliary parameter, and $N$ is a nonlinear operator given by

$$
N[\phi(\tau ; p)]=a_{1} \frac{\partial^{2} \phi}{\partial \tau^{2}}+a_{2} \frac{\partial \phi}{\partial \tau}+a_{3} \phi-\int_{-1}^{\tau} k(\tau, t) \phi(t) d t
$$

Differentiating (3.2) $m$ times with respect to the embedding parameter $p$ and then setting $p=0$ and finally dividing them by $m$ !, we have the so-called $m$ th-order deformation equation

$$
L\left[u_{m}(\tau)-\chi_{m} u_{m-1}(\tau)\right]=h R_{m}
$$

subject to boundary conditions

$$
u_{m}(-1)=u_{m}(1)=0
$$

where

$$
R_{m}(\tau)=a_{1} u_{m-1}^{\prime \prime}+a_{2} u_{m-1}^{\prime}+a_{3} u_{m-1}-g(\tau)\left(1-x_{m}\right)-\int_{-1}^{\tau} k(\tau, t) u_{m-1}(t) d t
$$

Applying the Chebyshev pseudospectral transformation on (3.15)-(3.17) results in

$$
\mathbf{A} \mathbf{U}_{m}=\left(x_{m}+h\right) \mathbf{A} \mathbf{U}_{m-1}-h\left[\mathbf{S}_{m-1}+\left(1-x_{m}\right) \mathbf{G}\right]
$$

subject to the boundary conditions

$$
u_{m}\left(\tau_{0}\right)=u_{m}\left(\tau_{N}\right)=0
$$

where $\mathbf{A}$ and $\mathbf{G}$ are as defined in (3.10) and

$$
\mathbf{U}_{m}=\left[u_{m}\left(\tau_{0}\right), u_{m}\left(\tau_{1}\right), \ldots, u_{m}\left(\tau_{N}\right)\right]^{T}, \quad \mathbf{S}_{m-1}=\int_{-1}^{\tau} k(\tau, t) \mathbf{U}_{m-1} d t
$$

To implement the boundary conditions we delete the first and the last rows of $\mathbf{S}_{m-1}$ and the first and the last rows and columns of $\mathbf{A}$.

Finally, this recursive formula can be written as following for $m \geq 1$ :

$$
\mathbf{U}_{m}=\left(\chi_{m}+h\right) \mathbf{A}^{-1} \mathbf{A} \mathbf{U}_{m-1}-h \mathbf{A}^{-1}\left[\mathbf{S}_{m-1}+\left(1-\chi_{m}\right) \mathbf{G}\right]
$$


so, starting from the initial approximation. We can obtain higher-order approximation $\mathbf{U}_{m}$ for $m \geq 1$, recursively.

\section{Calculation of $\mathbf{S}_{m-1}$}

Consider the well-known Chebyshev polynomials of first kind of degree $n$, defined by [25]

$$
T_{n}(x)=\cos \left(n \cos ^{-1}(x)\right), \quad n \geq 0
$$

Also they are derived from the following recursive formula:

$$
T_{0}(x)=1, \quad T_{1}(x)=x, \quad T_{n+1}(x)=2 x T_{n}(x)-T_{n-1}(x), \quad n=1,2,3, \ldots
$$

In this section we approximate the integrand in (3.15) by letting

$$
k\left(\tau_{m}, t, y_{m-1}(t)\right)=\sum_{j=0}^{m-1} T_{j}(t) w_{m j}, \quad t=\tau_{0}, \tau_{1}, \ldots, \tau_{m-1}, \quad m=1,2, \ldots, N
$$

where $T_{j}(t), j=0,1, \ldots, m-1$ are the basis functions.

Clearly, $w_{m j}, 0 \leq j \leq m-1$ can be obtained by solving the following matrix equation:

$$
\left(\begin{array}{cccc}
T_{0}\left(\tau_{0}\right) & T_{1}\left(\tau_{0}\right) & \ldots & T_{m-1}\left(\tau_{0}\right) \\
T_{0}\left(\tau_{1}\right) & T_{1}\left(\tau_{1}\right) & \ldots & T_{m-1}\left(\tau_{1}\right) \\
\vdots & \vdots & & \vdots \\
T_{0}\left(\tau_{m-1}\right) & T_{1}\left(\tau_{m-1}\right) & \ldots & T_{m-1}\left(\tau_{m-1}\right)
\end{array}\right)\left(\begin{array}{c}
w_{m, 0} \\
w_{m, 1} \\
\vdots \\
w_{m, m-1}
\end{array}\right)=\left(\begin{array}{c}
k\left(t_{m}, \tau_{0}, y_{0}\right) \\
k\left(t_{m}, \tau_{1}, y_{1}\right) \\
\vdots \\
k\left(t_{m}, \tau_{m-1}, y_{m-1}\right)
\end{array}\right)
$$

where $\tau_{j}=t_{j}, j=0,1, \ldots, m-1$ are Chebyshev collocation points defined in (2.6). We can obtain $w_{m j}$ by solving the above system so the integral in (3.15) can be rewritten as following:

$$
\int_{-1}^{\tau} k(\tau, t) y_{m-1}(t) d t=\sum_{j=0}^{m-1} a_{m, j} w_{m, j}
$$

where

$$
a_{m, j}=\int_{-1}^{\tau} T_{j}(t) d t=P T_{j}(\tau)
$$


Table 1: Numerical result of Example 5.1 against the HAM and the SHAM solutions whit $h=-0.85$.

\begin{tabular}{lccccccc}
\hline$x$ & \multicolumn{5}{c}{ SHAM } & \multicolumn{5}{c}{ HAM } \\
& 2nd order & 4th order & 5th order & 2nd order & 5th order & 11th order & Numerical \\
\hline-0.8 & -0.360376 & -0.360001 & -0.36000000 & -0.370525 & -0.360086 & -0.36000000 & -0.36000000 \\
-0.6 & -0.640699 & -0.640003 & -0.64000000 & -0.658320 & -0.640123 & -0.64000000 & -0.64000000 \\
-0.4 & -0.840976 & -0.840005 & -0.84000000 & -0.860438 & -0.840105 & -0.84000000 & -0.84000000 \\
-0.2 & -0.961198 & -0.960005 & -0.96000000 & -0.979684 & -0.960078 & -0.96000000 & -0.96000000 \\
0.0 & -1.001354 & -1.000005 & -1.00000000 & -1.018938 & -1.000066 & -1.00000000 & -1.00000000 \\
0.2 & -0.961421 & -0.960005 & -0.96000000 & -0.978828 & -0.960067 & -0.96000000 & -0.96000000 \\
0.4 & -0.841370 & -0.840005 & -0.84000000 & -0.857858 & -0.840073 & -0.84000000 & -0.84000000 \\
0.6 & -0.641159 & -0.640004 & -0.64000000 & -0.654041 & -0.64048 & -0.64000000 & -0.64000000 \\
0.8 & -0.360727 & -0.360002 & -0.36000000 & -0.367054 & -0.360011 & -0.36000000 & -0.36000000 \\
\hline
\end{tabular}

where $P$ as in [26], that is the $(N+1) \times(N+1)$ operational matrix for integration as follows:

$$
\mathbf{P}=\left(\begin{array}{ccccccc}
1 & 1 & 0 & 0 & \ldots & 0 & 0 \\
-\frac{1}{4} & 0 & -\frac{1}{4} & 0 & \ldots & 0 & 0 \\
-\frac{1}{3} & -\frac{1}{2} & 0 & \frac{1}{6} & \ldots & 0 & 0 \\
\frac{1}{8} & 0 & -\frac{1}{4} & 0 & \ldots & 0 & 0 \\
\vdots & \vdots & \vdots & \vdots & \ddots & \vdots & \vdots \\
\frac{(-1)^{N}}{(N-1)^{2}-1} & 0 & 0 & 0 & \ldots & 0 & \frac{1}{2 N} \\
\frac{(-1)^{N+1}}{N^{2}-1} & 0 & 0 & 0 & \ldots & -\frac{1}{2 N-2} & 0
\end{array}\right)
$$

\section{Numerical Examples}

In this section we apply the technique described in Section 3 to some illustrative examples of second-order Volterra integrodifferential equations.

Example 5.1. Consider the second-order Volterra integrodifferential equation

$$
y^{\prime \prime}(x)-x y^{\prime}(x)+x y(x)=\frac{x^{4}}{6}+x^{3}-2 x^{2}-\frac{x}{3}+\frac{5}{2}+\int_{-1}^{x}(x-2 t) y(t) d t
$$

subject to the boundary conditions $y(-1)=y(1)=0$ with the exact solution $y(x)=x^{2}-1$.

We employ HAM and SHAM to solve this example. In Table 1, there is a comparison of the numerical result against the HAM and SHAM approximation solutions at different orders. It is worth noting that the SHAM results become very highly accurate only with a few iterations and fourth-order results become very close to the exact solution. Comparison of the numerical solution with the 4th-order SHAM solution for $h=-0.85$ is made in Figure 1. It 


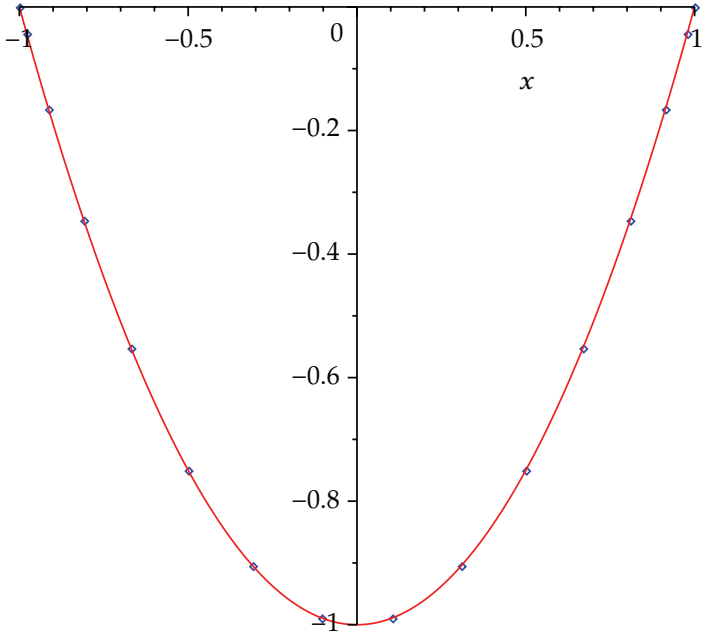

- Exact solution

$y_{4}$

Figure 1: Comparison of the numerical solution with the 4th-order SHAM solution for $h=-0.85$.

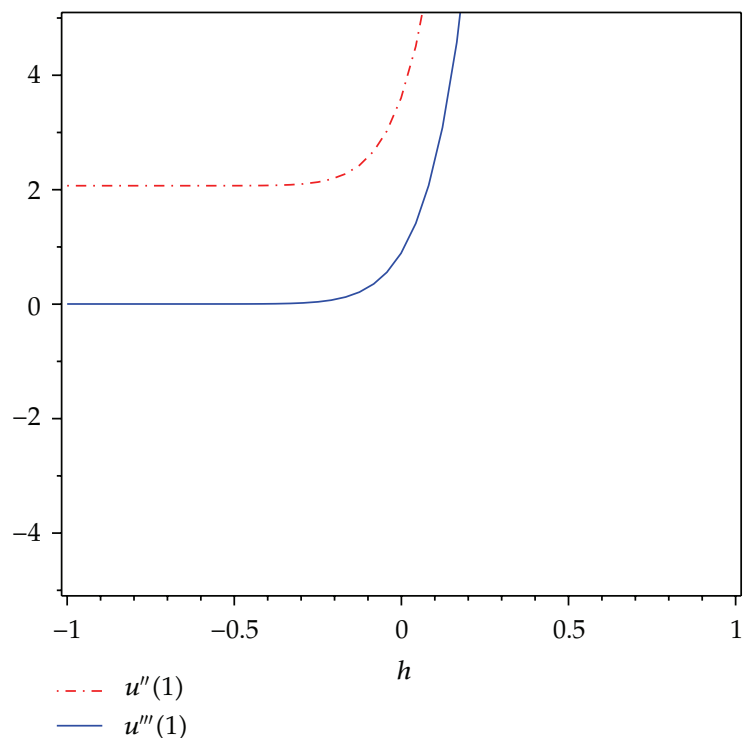

Figure 2: 4th-order SHAM $h$-curve.

can be seen from the $h$-curves (Figures 2 and 3) that if $-1 \leq h \leq-0.2$ and $-1 \leq h \leq-0.4$ then the SHAM an HAM approximate solutions are in well agreement with the exact solution. In Table 2, we present that the SHAM results for another value of $h$ in $h(h=-0.9)$, are also very highly accurate, and in sixth order we obtain the exact numerical solutions. In HAM we choose $y_{0}(x)=\left(x^{2}-1\right) / 2$ as initial guess. 


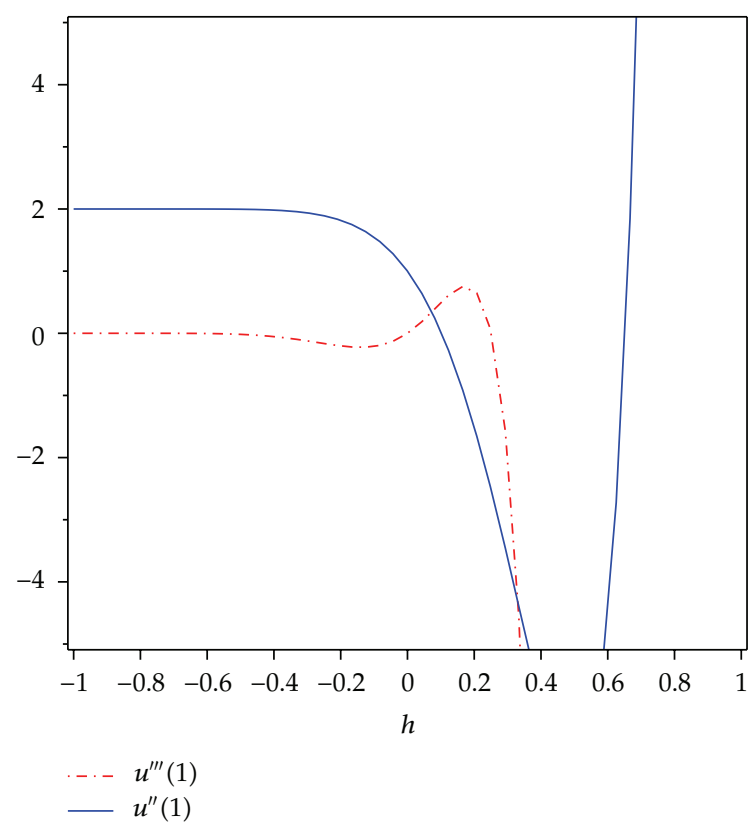

Figure 3: 11th-order HAM $h$-curve.

Table 2: Numerical result of Example 5.1 against the SHAM solutions whit $h=-0.9$.

\begin{tabular}{lcccc}
\hline$x$ & 5th order & 6th order & 7th order & Numerical \\
\hline-0.8 & -0.35999595 & -0.36000000 & -0.36000000 & -0.36000000 \\
-0.6 & -0.63999432 & -0.64000000 & -0.64000000 & -0.64000000 \\
-0.4 & -0.83999202 & -0.84000000 & -0.84000000 & -0.84000000 \\
-0.2 & -0.95999009 & -0.96000000 & -0.96000000 & -0.96000000 \\
0.0 & -0.99998868 & -1.00000000 & -1.00000000 & -1.00000000 \\
0.2 & -0.95998890 & -0.96000000 & -0.96000000 & -0.96000000 \\
0.4 & -0.83998833 & -0.84000000 & -0.84000000 & -0.84000000 \\
0.6 & -0.63999070 & -0.64000000 & -0.64000000 & -0.64000000 \\
0.8 & -0.35999373 & -0.36000000 & -0.36000000 & -0.36000000 \\
\hline
\end{tabular}

Example 5.2. Consider the second-order Volterra integrodifferential equation

$$
y^{\prime \prime}(x)+y(x)=-4 x^{6}+6 x^{4}+x^{3}+5 x-2+\int_{-1}^{x} 24 t^{2} y(t) d t
$$

subject to the boundary conditions $y(-1)=y(1)=0$ with the exact solution $y(x)=x^{3}-x$.

Similar to the previous example, we employ HAM and SHAM to solve this example. In Table 3, there is a comparison of the numerical results against the HAM and SHAM approximation solutions (5.2) at different orders. It is worth noting that the rate of convergence in SHAM results is higher than the HAM results. Comparison of the numerical solution with the 16th-order SHAM solution for $h=-0.4$ is made in Figure 4 . It can be seen from the $h$-curves (Figures 5 and 6) that if $-0.4 \leq h \leq 0$ and $-0.6 \leq h \leq-0.3$ then 
Table 3: Numerical result of Example 5.2 against the HAM and the SHAM solutions whit $h=-0.4$.

\begin{tabular}{|c|c|c|c|c|c|c|}
\hline \multirow{2}{*}{$x$} & \multicolumn{3}{|c|}{ SHAM } & \multirow{2}{*}{ Numerical } & \multicolumn{2}{|c|}{ HAM } \\
\hline & 8th order & 14 th order & 16th order & & 8th order & 23th order \\
\hline $\begin{array}{l}-0.8 \\
\end{array}$ & 0.28818730 & 0.28800272 & 0.28800000 & 0.28800000 & 0.28786022 & 0.28800097 \\
\hline-0.6 & 0.38426358 & 0.38400174 & 0.38400000 & 0.38400000 & 0.38548670 & 0.3840003 \\
\hline-0.4 & 0.33618044 & 0.33599640 & 0.33600000 & 0.33600000 & 0.34009630 & 0.33600088 \\
\hline-0.2 & 0.19199056 & 0.19198946 & 0.19200000 & 0.19200000 & 0.19905046 & 0.1920014 \\
\hline 0.0 & -0.00024441 & -0.00000165 & 0.00000000 & 0.00000000 & -0.00041662 & 0.00000166 \\
\hline 0.2 & -0.19249771 & -0.19202092 & -0.19200000 & -0.19200000 & -0.18206491 & -0.19209884 \\
\hline 0.4 & -0.33673440 & -0.33602266 & -0.33600000 & -0.33600000 & -0.32825115 & -0.33600119 \\
\hline 0.6 & -0.36072793 & -0.38401942 & -0.38400000 & -0.38400000 & -0.38049994 & -0.38400153 \\
\hline 0.8 & -0.28863110 & -0.28801009 & -0.28800000 & -0.28800000 & -0.28821582 & $-0.2880015 \mathrm{c}$ \\
\hline
\end{tabular}

the approximate solution is in well agreement with the exact solution. In HAM, we choose $y_{0}(x)=-x^{2}+1$ as initial guess.

Example 5.3. Consider the first-order Fredholm integrodifferential equation [27]

$$
y^{\prime}(x)-y(x)=\sin (4 \pi x)-\cos (2 \pi x)-2 \pi \sin (2 \pi x)-\int_{-1}^{1} \sin (4 \pi x+2 \pi t) y(t) d t
$$

subject to the boundary conditions $y(-1)=y(1)=1$.

In this example, by a change of variables, the Fredholm-integro differential equation with nonhomogeneous conditions are transformed to the equation with homogeneous conditions and then apply the spectral homotopy analysis method for this problem. According to governing equation and the boundary condition it is reasonable to set the transformation

$$
y(x)=\cos (2 \pi x)+Y(x)
$$

to make the governing boundary conditions homogeneous. Substituting (5.4) in (5.3) and boundary conditions gives

$$
Y^{\prime}(x)-Y(x)=\int_{-1}^{1} \sin (4 \pi x+2 \pi t) Y(t) d t
$$

subject to the boundary condition

$$
Y(-1)=Y(1)=0
$$

Comparison between absolute errors in solutions by SHAM and Legendre collocation matrix method (LCMM) is tabulated in Table 4. It is worth noting that the SHAM results become very highly accurate only with four iterations. 


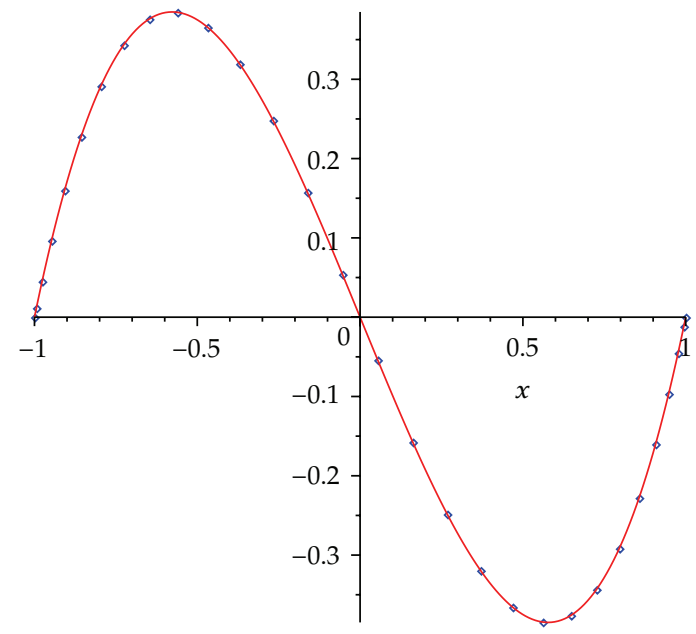

- Exact solution

- $y_{16}$

Figure 4: Comparison of the numerical solution with the 16th-order SHAM solution for $h=-0.4$.

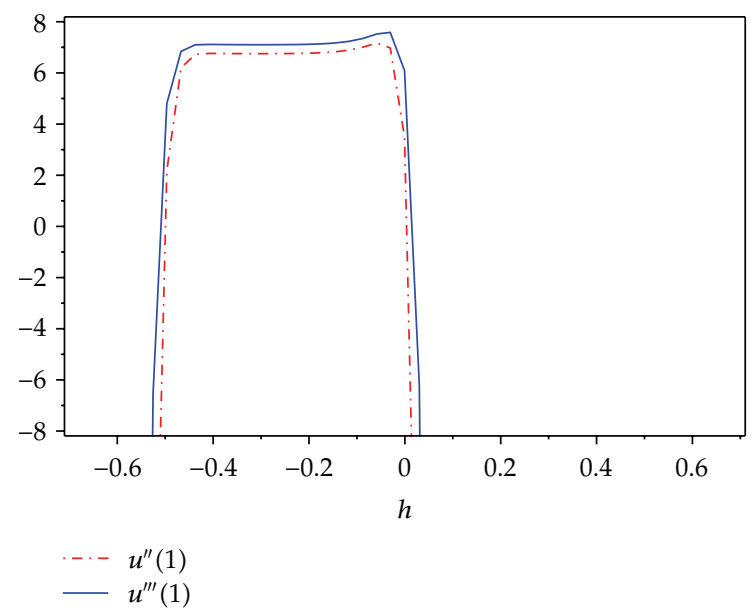

Figure 5: 16th-order SHAM $h$-curve3.

Example 5.4. Consider the following second-order Fredholm integrodifferential equation [28]:

$$
y^{\prime \prime}(x)+x y^{\prime}(x)-x y(x)=e^{x}-2 \sin (x)+\int_{-1}^{1} \sin (x) e^{-t} y(t) d t
$$

where $y(-1)=e^{-1}, y(1)=e$.

In this example, by a change of variables, the Fredholm-integro differential equation with nonhomogeneous conditions are transformed to the equation with homogeneous conditions and then we apply the spectral homotopy analysis method for this problem. 


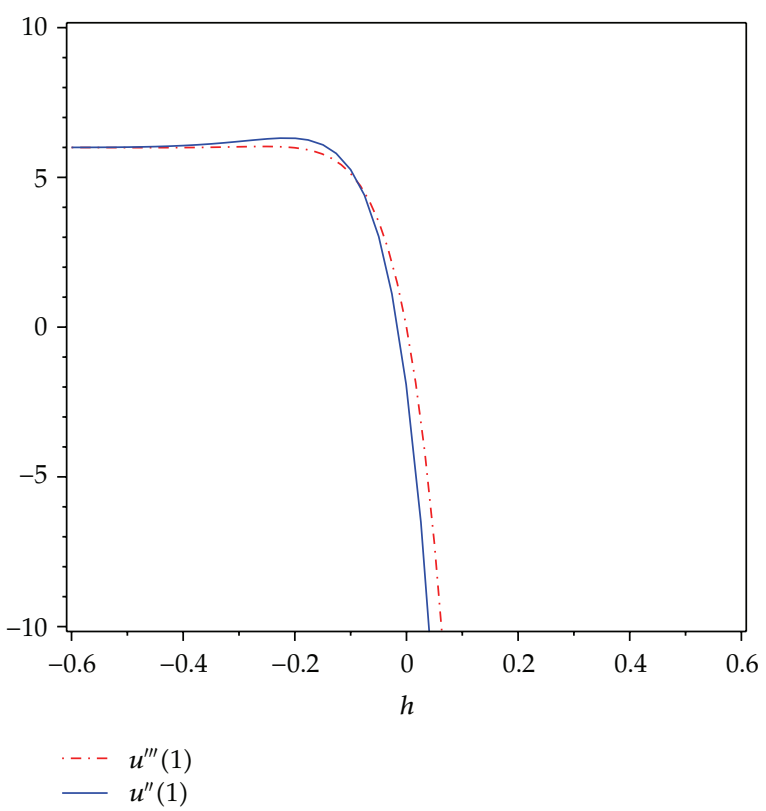

Figure 6: 23th-order HAM $h$-curve.

Table 4: Absolute errors for Example 5.3.

\begin{tabular}{lcc}
\hline$x$ & SHAM & LCMM \\
\hline-1.0 & $3.30 \times 10^{-19}$ & $2.49 \times 10^{0}$ \\
-0.8 & $1.73 \times 10^{-9}$ & $3.56 \times 10^{-1}$ \\
-0.6 & $6.69 \times 10^{-9}$ & $2.66 \times 10^{-1}$ \\
-0.4 & $2.91 \times 10^{-10}$ & $5.85 \times 10^{-2}$ \\
-0.2 & $6.93 \times 10^{-9}$ & $1.74 \times 10^{-2}$ \\
0.0 & $9.16 \times 10^{-9}$ & 0.00 \\
0.2 & $6.93 \times 10^{-9}$ & $1.97 \times 10^{-2}$ \\
0.4 & $2.91 \times 10^{-10}$ & $7.50 \times 10^{-2}$ \\
0.6 & $6.69 \times 10^{-9}$ & $2.59 \times 10^{-2}$ \\
0.8 & $1.73 \times 10^{-9}$ & $4.16 \times 10^{-1}$ \\
1.0 & $6.38 \times 10^{-17}$ & $2.58 \times 10^{0}$ \\
\hline
\end{tabular}

According to governing equation and the boundary condition it is reasonable to set the transformation

$$
y(x)=e^{x}+Y(x)
$$

to make the governing boundary conditions homogeneous. Substituting (5.8) in (5.7) and boundary conditions gives

$$
Y^{\prime \prime}(x)+x Y^{\prime}(x)-x Y(x)=\int_{-1}^{1} \sin (x) e^{-t} Y(t) d t
$$


Table 5: Absolute errors for Example 5.4.

\begin{tabular}{lcc}
\hline$x$ & $\begin{array}{c}\text { SHAM } \\
\text { 4th order }\end{array}$ & $\begin{array}{c}\text { HAM } \\
20 \text { th order }\end{array}$ \\
\hline 0.0 & 0 & 0 \\
0.2 & 0 & $2.2760 \times 10^{-14}$ \\
0.4 & 0 & $3.5971 \times 10^{-13}$ \\
0.6 & $1 \times 10^{-19}$ & $1.8209 \times 10^{-12}$ \\
0.8 & $1 \times 10^{-19}$ & $5.7547 \times 10^{-12}$ \\
1.0 & 0 & $1.4550 \times 10^{-11}$ \\
1.2 & $1.2241 \times 10^{-15}$ & $2.9134 \times 10^{-11}$ \\
1.4 & $1.1239 \times 10^{-13}$ & $5.3974 \times 10^{-11}$ \\
\hline
\end{tabular}

subject to boundary condition

$$
Y(-1)=Y(1)=0
$$

Comparison between absolute errors in solutions by SHAM and HAM is tabulated in Table 5. It is worth noting that the SHAM results become very highly accurate only with four iterations.

\section{Conclusion}

In this paper for the first time in the literature we presented the application of spectral homotopy analysis method (SHAM) in solving linear integro differential equations. A comparison was made between exact analytic solutions and numerical results from the spectral homotopy analysis method and homotopy analysis method (HAM) solutions. The numerical results indicate that in SHAM the rate of convergence is faster than HAM. For example, in Example 5.1 we found that the fourth-order SHAM approximation sufficiently gives a match with the numerical results up to twelve decimal places.In contrast, HAM solutions have a good agreement with the numerical results in 11th-order. In this paper we choose the admissible value of $h$ from $h$-curve for $y^{\prime \prime}(1)$ and $y^{\prime \prime \prime}(1)$. As it is shown in Figures 2 and 3 the range of admissible $h$ values is much wider in SHAM than in HAM. In using HAM there is restriction on choosing an initial approximation that the higher-order deformation can be integrated whereas in SHAM the initial approximation is the solution of the nonhomogeneous linear part of (3.1). The spectral homotopy analysis method is more efficient as it does not depend on the rule of coefficient ergodicity unlike the HAM. With some changes we can apply this method on Fredholm integrodifferential equations. In Example 5.3 [27], we compare the SHAM solution with Legendre collocation matrix method (LCCM) to solve linear Fredholm integrodifferential equation. As can be seen in Table 4 SHAM is more accurate and efficient than LCCM. In this work we obtained the numerical results up to twelve decimal places having well agreement with exact solution but reported them only up to six decimal places.

In this paper, we described the spectral homotopy analysis method to solve linear Volterra and Fredholm integrodifferential equations; however, it remains to be generalized and verified for more complicated integral equations that we consider it as future works. 


\section{Acknowledgment}

The authors gratefully acknowledge that this research was partially supported by the Universiti Putra Malaysia under the ERGS Grant Scheme having project number 5527068.

\section{References}

[1] L. K. Forbes, S. Crozier, and D. M. Doddrell, "Calculating current densities and fields produced by shielded magnetic resonance imaging probes," SIAM Journal on Applied Mathematics, vol. 57, no. 2, pp. 401-425, 1997.

[2] K. Parand, S. Abbasbandy, S. Kazem, and J. A. Rad, "A novel application of radial basis functions for solving a model of first-order integro-ordinary differential equation," Communications in Nonlinear Science and Numerical Simulation, vol. 16, no. 11, pp. 4250-4258, 2011.

[3] P. Darania and A. Ebadian, "A method for the numerical solution of the integro-differential equations," Applied Mathematics and Computation, vol. 188, no. 1, pp. 657-668, 2007.

[4] A. Karamete and M. Sezer, "A Taylor collocation method for the solution of linear integro-differential equations," International Journal of Computer Mathematics, vol. 79, no. 9, pp. 987-1000, 2002.

[5] S. J. Liao, The proposed homotopy analysis technique for the solution of nonlinear problems [Ph.D. thesis], Shanghai Jiao Tong University, 1992.

[6] A. M. Lyapunov, The General Problem of the Stability of Motion, Taylor \& Francis, London, UK, 1992.

[7] G. Adomian, "A review of the decomposition method and some recent results for nonlinear equations," Mathematical and Computer Modelling, vol. 13, no. 7, pp. 17-43, 1990.

[8] G. Adomian and R. Rach, "Noise terms in decomposition solution series," Computers E Mathematics with Applications, vol. 24, no. 11, pp. 61-64, 1992.

[9] G. Adomian and R. Rach, "Analytic solution of nonlinear boundary value problems in several dimensions by decomposition," Journal of Mathematical Analysis and Applications, vol. 174, no. 1, pp. 118-137, 1993.

[10] G. Adomian, Solving Frontier Problems of Physics: The Decomposition Method, vol. 60, Kluwer Academic, Boston, Mass, USA, 1994.

[11] P. K. Bera and J. Datta, "Linear delta expansion technique for the solution of anharmonic oscillations," Pramana Journal of Physics, vol. 68, no. 1, pp. 117-122, 2007.

[12] S. Liao, "On the homotopy analysis method for nonlinear problems," Applied Mathematics and Computation, vol. 147, no. 2, pp. 499-513, 2004.

[13] J. H. He, "The homotopy perturbation method for nonlinear oscillators with discontinuities," Applied Mathematics and Computation, vol. 151, no. 1, pp. 287-292, 2004.

[14] G. Domairry, A. Mohsenzadeh, and M. Famouri, "The application of homotopy analysis method to solve nonlinear differential equation governing Jeffery-Hamel flow," Communications in Nonlinear Science and Numerical Simulation, vol. 14, no. 1, pp. 85-95, 2009.

[15] Z. Ziabakhsh and G. Domairry, "Solution of the laminar viscous flow in a semi-porous channel in the presence of a uniform magnetic field by using the homotopy analysis method," Communications in Nonlinear Science and Numerical Simulation, vol. 14, no. 4, pp. 1284-1294, 2009.

[16] J.-H. He, "Homotopy perturbation technique," Computer Methods in Applied Mechanics and Engineering, vol. 178, no. 3-4, pp. 257-262, 1999.

[17] T. Hayat, S. Noreen, and M. Sajid, "Heat transfer analysis of the steady flow of a fourth grade fluid," International Journal of Thermal Sciences, vol. 47, no. 5, pp. 591-599, 2008.

[18] M. Jalaal, D. D. Ganji, and G. Ahmadi, "Analytical investigation on acceleration motion of a vertically falling spherical particle in incompressible Newtonian media," Advanced Powder Technology, vol. 21, no. 3, pp. 298-304, 2010.

[19] D. D. Ganji, G. A. Afrouzi, and R. A. Talarposhti, "Application of variational iteration method and homotopy-perturbation method for nonlinear heat diffusion and heat transfer equations," Physics Letters, Section A, vol. 368, no. 6, pp. 450-457, 2007.

[20] S. Abbasbandy, "Modified homotopy perturbation method for nonlinear equations and comparison with Adomian decomposition method," Applied Mathematics and Computation, vol. 172, no. 1, pp. 431438, 2006.

[21] S. S. Motsa, P. Sibanda, and S. Shateyi, "A new spectral-homotopy analysis method for solving a nonlinear second order BVP," Communications in Nonlinear Science and Numerical Simulation, vol. 15, no. 9, pp. 2293-2302, 2010. 
[22] S. S. Motsa, P. Sibanda, F. G. Awad, and S. Shateyi, "A new spectral-homotopy analysis method for the MHD Jeffery-Hamel problem," Computers \& Fluids, vol. 39, no. 7, pp. 1219-1225, 2010.

[23] S. S. Motsa and P. Sibanda, "A new algorithm for solving singular IVPs of Lane-Emden type," in Proceedings of the 4th International Conference on Applied Mathematics, Simulation, Modelling (ASM '10), pp. 176-180, Corfu Island, Greece, July 2010.

[24] W. S. Don and A. Solomonoff, "Accuracy and speed in computing the Chebyshev collocation derivative," SIAM Journal on Scientific Computing, vol. 16, no. 6, pp. 1253-1268, 1995.

[25] L. M. Delves and J. L. Mohamed, Computational Methods for Integral Equations, Cambridge University Press, Cambridge, UK, 1985.

[26] K. Maleknejad, S. Sohrabi, and Y. Rostami, "Numerical solution of nonlinear Volterra integral equations of the second kind by using Chebyshev polynomials," Applied Mathematics and Computation, vol. 188, no. 1, pp. 123-128, 2007.

[27] S. Yalçinbaş, M. Sezer, and H. H. Sorkun, "Legendre polynomial solutions of high-order linear Fredholm integro-differential equations," Applied Mathematics and Computation, vol. 210, no. 2, pp. 334-349, 2009.

[28] H. M. Jaradat, F. Awawdeh, and O. Alsayyed, "Series solution to the high-order integro-differential equations," Fascicola Matematica, vol. 16, pp. 247-257, 2009. 


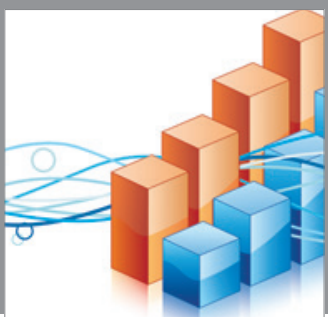

Advances in

Operations Research

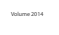

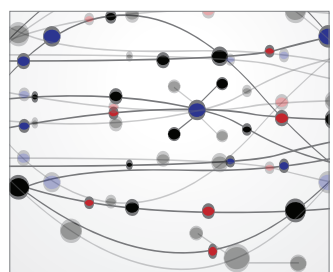

\section{The Scientific} World Journal
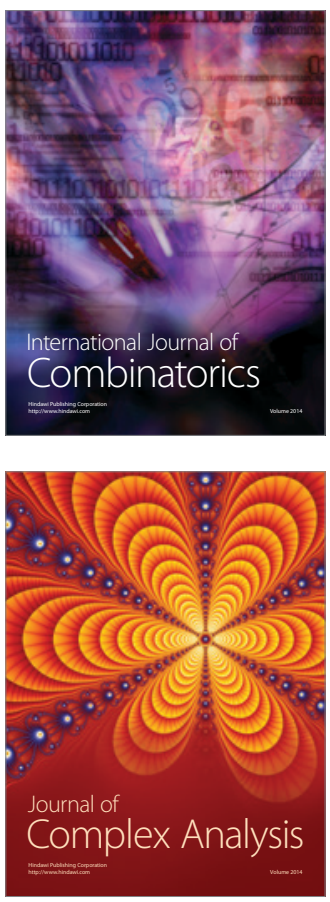

International Journal of

Mathematics and

Mathematical

Sciences
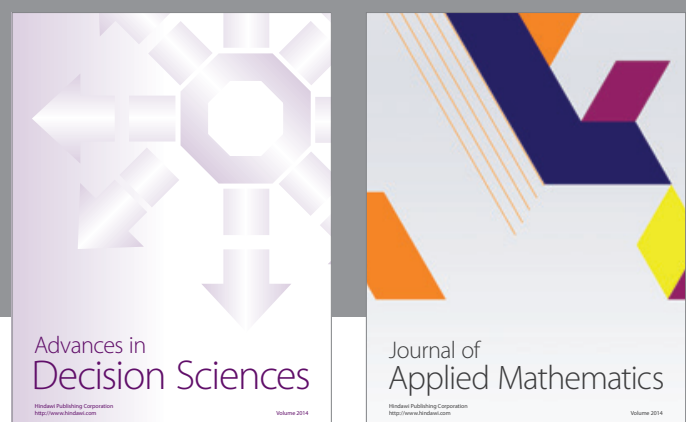

Journal of

Applied Mathematics
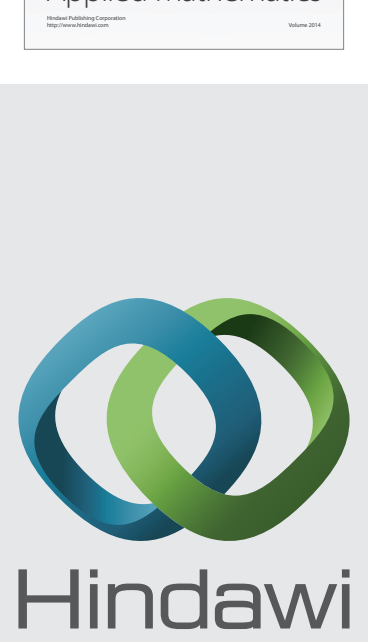

Submit your manuscripts at http://www.hindawi.com
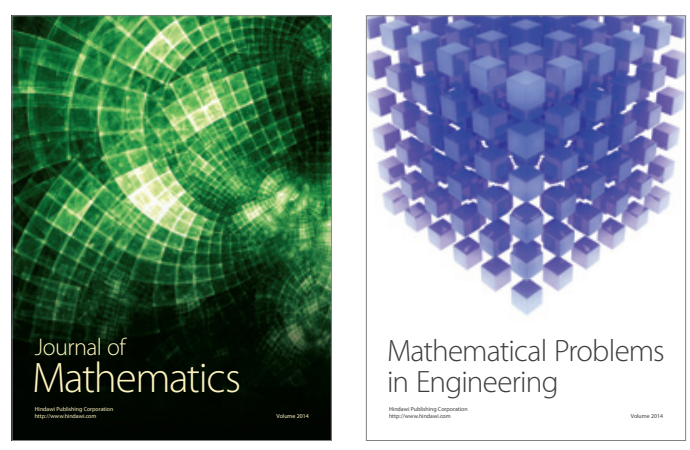

Mathematical Problems in Engineering
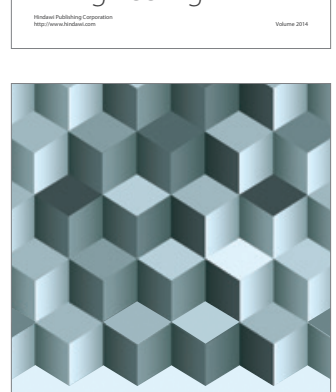

Journal of

Function Spaces
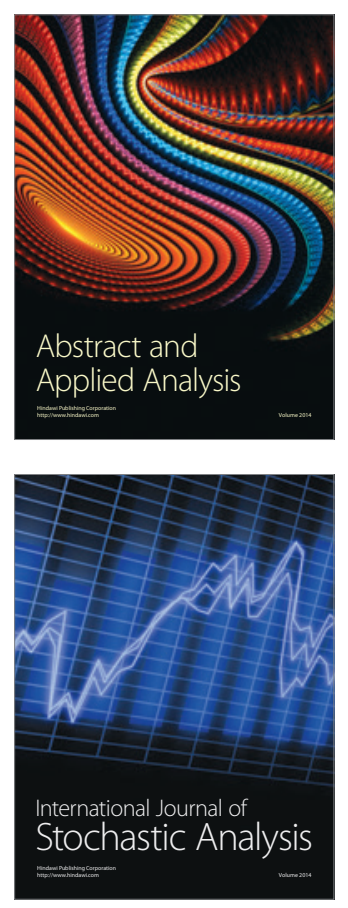

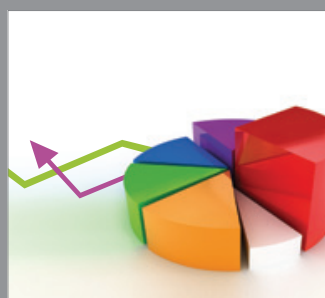

ournal of

Probability and Statistics

Promensencen
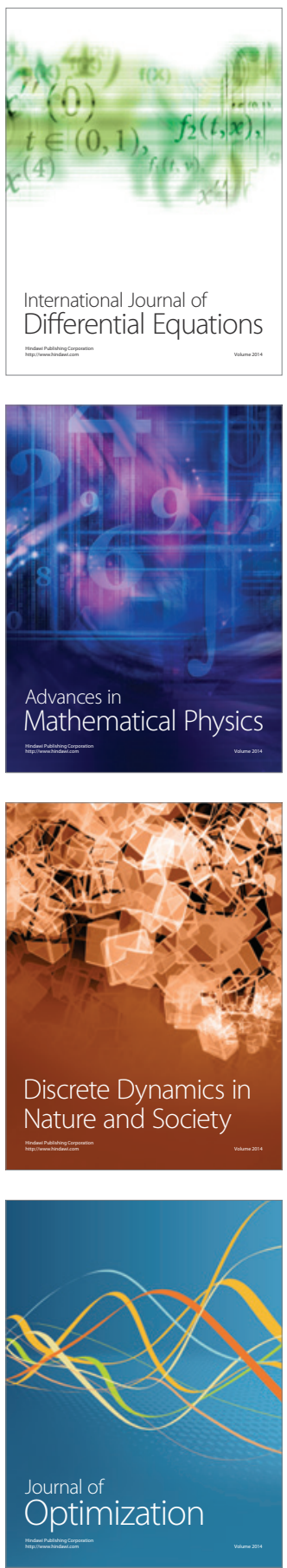